\title{
Study of the continuum limit of the Schwinger model using Wilson's lattice formulation
}

\section{Yuya Shimizu*}

RIKEN Advanced Institute for Computational Science, Kobe, Hyogo 650-0047, Japan

\section{Yoshinobu Kuramashi}

Graduate School of Pure and Applied Sciences, University of Tsukuba,

Tsukuba, Ibaraki 305-8571, Japan

Center for Computational Sciences, University of Tsukuba, Tsukuba, Ibaraki 305-8577, Japan

RIKEN Advanced Institute for Computational Science, Kobe, Hyogo 650-0047, Japan

\begin{abstract}
We estimate the critical point of the Schwinger model in the $\theta$ vaccum by taking the continuum limit of Wilson's lattice formulation. The decorated tensor renormalization group which has been recently proposed by Dittrich et al. is employed for numerical investigation. We compare our numerical results with those of Byrnes et al., which were derived from Kogut-Susskind's lattice formulation employing the density matrix renormalization group.
\end{abstract}

The 33rd International Symposium on Lattice Field Theory

14-18 July 2015

Kobe International Conference Center, Kobe, Japan

\footnotetext{
* Speaker.
} 


\section{Lattice Schwinger model in the Wilson formulation}

We here employ the path-integral formulation of the lattice Schwinger model with one flavor of the Wilson fermion:

$$
Z=\int \mathscr{D} U \operatorname{det} D[U] e^{-S_{g}[U]} .
$$

The Wilson-Dirac matrix is given by

$$
\begin{aligned}
\bar{\psi} D[U] \psi= & \frac{1}{2 \kappa} \sum_{n, \alpha} \bar{\psi}_{n, \alpha} \psi_{n, \alpha} \\
& -\frac{1}{2} \sum_{n, \mu, \alpha, \beta} \bar{\psi}_{n, \alpha}\left\{\left(1-\gamma_{\mu}\right)_{\alpha, \beta} U_{n, \mu} \psi_{n+\hat{\mu}, \beta}\right. \\
& \left.+\left(1+\gamma_{\mu}\right)_{\alpha, \beta} U_{n-\hat{\mu}, \mu}^{\dagger} \psi_{n-\hat{\mu}, \beta}\right\}
\end{aligned}
$$

and the plaquette gauge action including the $\theta$ term is

$$
\begin{gathered}
S_{g}=-\beta \sum_{p} \cos \varphi_{p}-i \theta Q, \\
Q=\frac{1}{2 \pi} \sum_{p} q_{p}, \\
q_{p}=\varphi_{p} \quad \bmod 2 \pi,
\end{gathered}
$$

where $\varphi_{p}$ is a phase of an $\mathrm{U}(1)$ plaquette variable and $\theta$ is fixed to $\pi$ throughout this paper. For the details of our notation, please refer to Ref. [1,2]

\section{Decorated tensor renormalization group}

The decorated tensor renormalization group (DTRG) was recently proposed by Dittrich et al. [3] as a variation of the tensor renormalization group method which is suitable for lattice models having local gauge invariance. In order to get a decorated tensor network form of the partition function, the gauge part of the Boltzmann weight is decomposed by the character expansion $[4,5]$ :

$$
\begin{aligned}
& \exp \left\{\beta \cos \varphi_{p}+i \frac{\theta}{2 \pi} q_{p}\right\} \\
& =\sum_{m_{p}=-\infty}^{\infty} e^{i m_{p} \varphi_{p}} \sum_{k=-\infty}^{\infty} I_{k}(\beta) \frac{2 \sin \left(\frac{\theta+2 \pi\left(m_{p}-k\right)}{2}\right)}{\theta+2 \pi\left(m_{p}-k\right)} \\
& \simeq \sum_{m_{p}=-N_{\mathrm{ce}}}^{N_{\mathrm{ce}}-1} e^{i m_{p} \varphi_{p}} \sum_{k=-\infty}^{\infty} I_{k}(\beta) \frac{2 \sin \left(\frac{\theta+2 \pi\left(m_{p}-k\right)}{2}\right)}{\theta+2 \pi\left(m_{p}-k\right)},
\end{aligned}
$$

where $I_{k}$ is the modified Bessel function and we truncate the series up to $N_{\mathrm{ce}}$-th terms. After the fermionic part is also decomposed by using the properties of Grassmann numbers, the partition function can be rewritten as

$$
Z=\sum_{i_{p}, j_{p}, k_{p}, \cdots} \int \sum_{i, j, k, \cdots} T_{n ; i, j, k, l}^{\left(i_{p}, j_{p}, k_{p}, l_{p}\right)} T_{n+\hat{1} ; m, o, i, p}^{\left(i_{p}, j_{p}, k_{p}, l_{p}\right)} T_{n+\hat{2} ; q, r, s, j}^{\left(i_{p}, j_{p}, k_{p}, l_{p}\right)} \cdots
$$




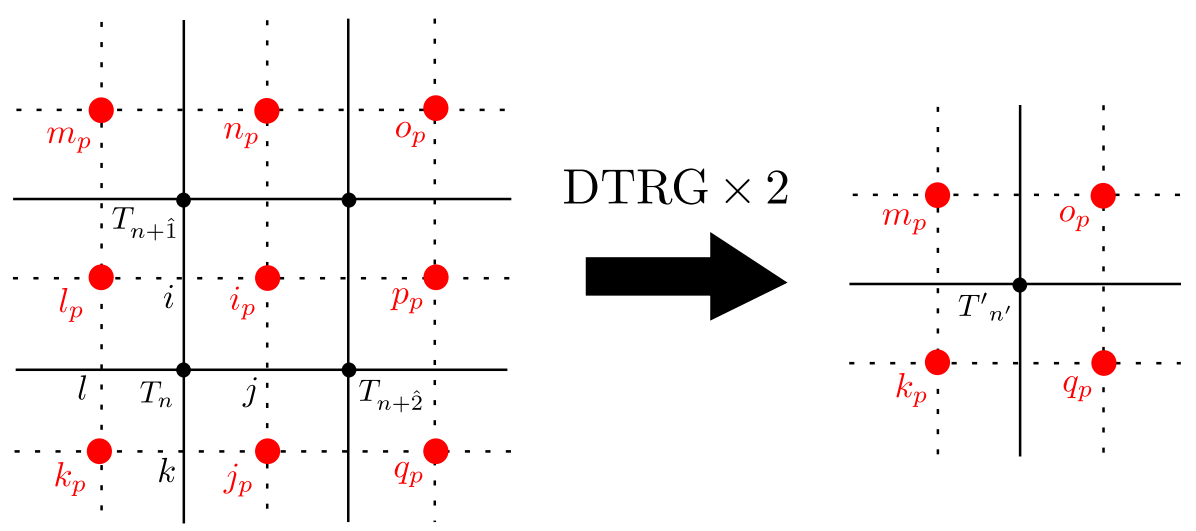

Figure 1: Decorated tensor network and its coarse-graining. Each tensor which sits on a lattice site is decorated by labels of the character expansion which surround the site. After iterations of the DTRG procedure, a part of labels $\left(i_{p}, j_{p}, l_{p}, n_{p}\right.$ and $p_{p}$ in the figure) are integrated out, but the others $\left(k_{p}, m_{p}, o_{p}\right.$ and $q_{p}$ in the figure) remain.

where $T^{\left(i_{p}, j_{p}, k_{p}, l_{p}\right)}$ is a Grassmann-valued tensor decorated by labels of the character expansion $\left\{i_{p}, j_{p}, k_{p}, l_{p}\right\}$, whose detail is given in Ref. [6]. Using the algorithm for two-dimensional systems described in Ref. [3], the decorated tensor network is coarse-grained and we get an approximate representation of the partition function. Then, each coarse-grained tensor has new indices which emerge from the singular value decomposition in the DTRG procedure, while keeping the decoration labels the same as their originals (See Figure 1.). Thanks to this property, the Gauss constraints are preserved under coarse-graining and that allows us to reduce computational cost remarkably. The details in case including fermions are discussed in Ref. [6].

\section{Numerical results}

To estimate the critical point at $\theta=\pi$, we calculate the following quantity:

$$
X \equiv \frac{\sum_{i_{p}} \sum_{i, j}(-1)^{i_{f} j_{f}} T_{n ; i, j, i, j}^{\left(i_{p}, i_{p}, i_{p}, i_{p}\right)}}{\sum_{i_{p}, j_{p}} \sum_{i, j, k, l}(-1)^{i_{f}+i_{f} j_{f}+k_{f} l_{f}} T_{n ; i, j, k, j}^{\left(i_{p}, j_{p}, j_{p}, i_{p}\right)} T_{n+\hat{1} ; k, l, i, l}^{\left(j_{p}, i_{p}, i_{p}, j_{p}\right)}},
$$

where $i_{f}=0\left(i_{f}=1\right)$ if the index $i$ corresponds to a bosonic (fermionic) state. This quantity was originally proposed by $\mathrm{Gu}$ and Wen [7] for bosonic cases. In the infinite volume (infrared) limit, it represents the degeneracy of the ground state. Figure 2 shows DTRG flows of $X$ for $\beta=10.0$. While the flow for $\kappa=0.24135$ reaches $X=1$, namely, the symmetric phase, the one for $\kappa=$ 0.24130 reaches $X=2$, the $Z(2)$-broken phase. Convergence values of $X$ for various $\kappa$ are given in Fig. 3. It clearly shows a phase transition in $0.24130<\kappa<0.24135$. It should be noted that there is a truncation error of the singular value decomposition in the DTRG procedure and we have to take care of its effect on our evaluation of $\kappa_{c}$. In this paper, we refer to the truncation number in each sector divided by decoration labels and Grassmann parity as $D$. Figure 4 shows the $D$ dependence of $\kappa_{c}$ evaluated from $X$. As you can see, $\kappa_{c}$ is converged well where $D \geq 15$ in this case. We also have a truncation error of the character expansion in Eq.(2.1). In Figs. 5, DTRG flows of $X$ for various $N_{\text {ce }}$ are presented. Fortunately, the higher-order terms seem not to contribute to the infinite 




Figure 2: DTRG flows of $X$ for $\beta=10.0, D=16$ and $N_{\text {ce }}=20$. The red symbols which converge to $X=2$ are the results for $\kappa=0.24130$ and the green ones which converge to $X=1$ are for $\kappa=0.24135$.

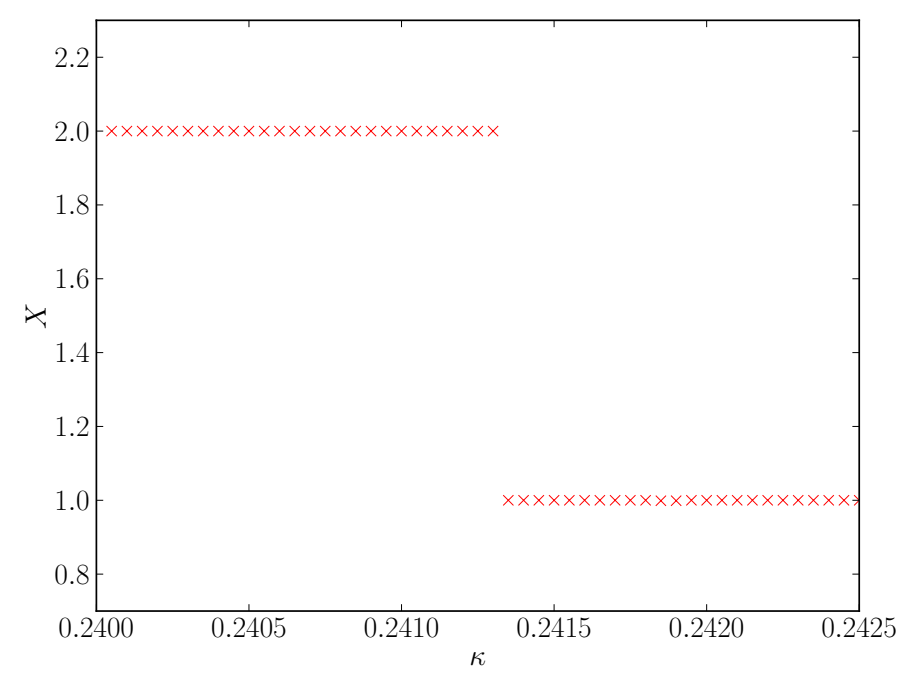

Figure 3: Convergence values of $X$ after 30 iterations of the DTRG procedure as a function of $\kappa$ for $\beta=10.0$, $D=16$ and $N_{\text {ce }}=20$. A phase transition is observed in $0.24130<\kappa<0.24135$.

volume limit. It means that the character expansion is quite powerful to investigate infrared physics. To estimate the continuum limit of the critical point, we perform the same analysis as above up to $\beta=700.0$. The continuum limit of the critical value of $\frac{m}{g}$ with $m=\frac{1}{2 \kappa}-2$ and $g=\frac{1}{\sqrt{\beta}}$ has been already evaluated by Byrnes et al. [8] using the Hamiltonian lattice formulation and the density matrix renormalization group technique. Although the lattice formulation we employ is different from that of Byrnes et al., our estimate should converge to the same value in the continuum limit because of the universality of the continuum limit. Figure 6 presents our results of $\frac{m}{g}$ from $\beta=10.0$ 


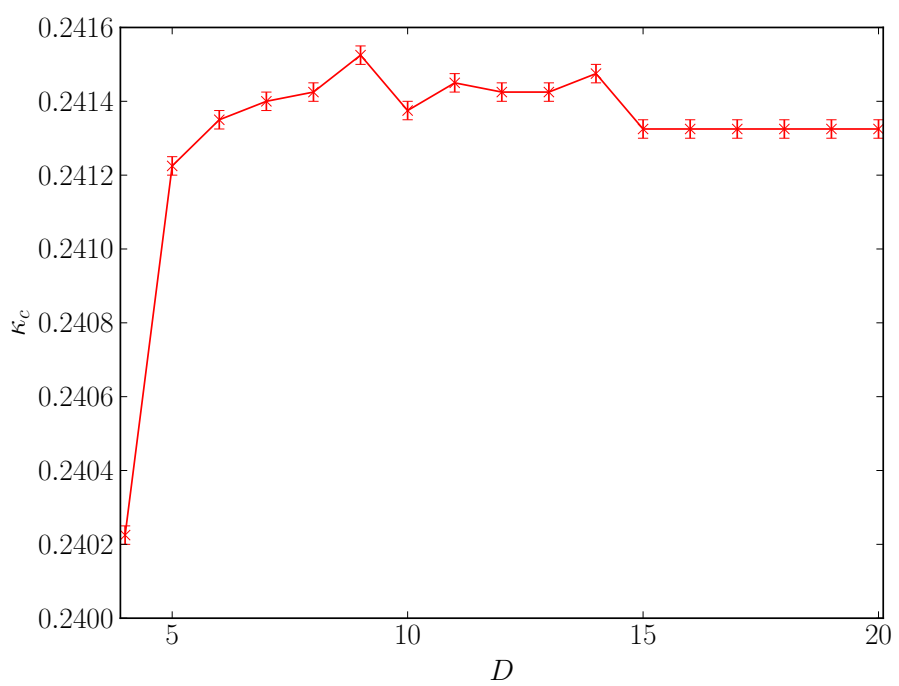

Figure 4: Convergence of $\kappa_{c}$ as a function of the truncation number $D$ for $\beta=10.0$ and $N_{\mathrm{ce}}=20$.
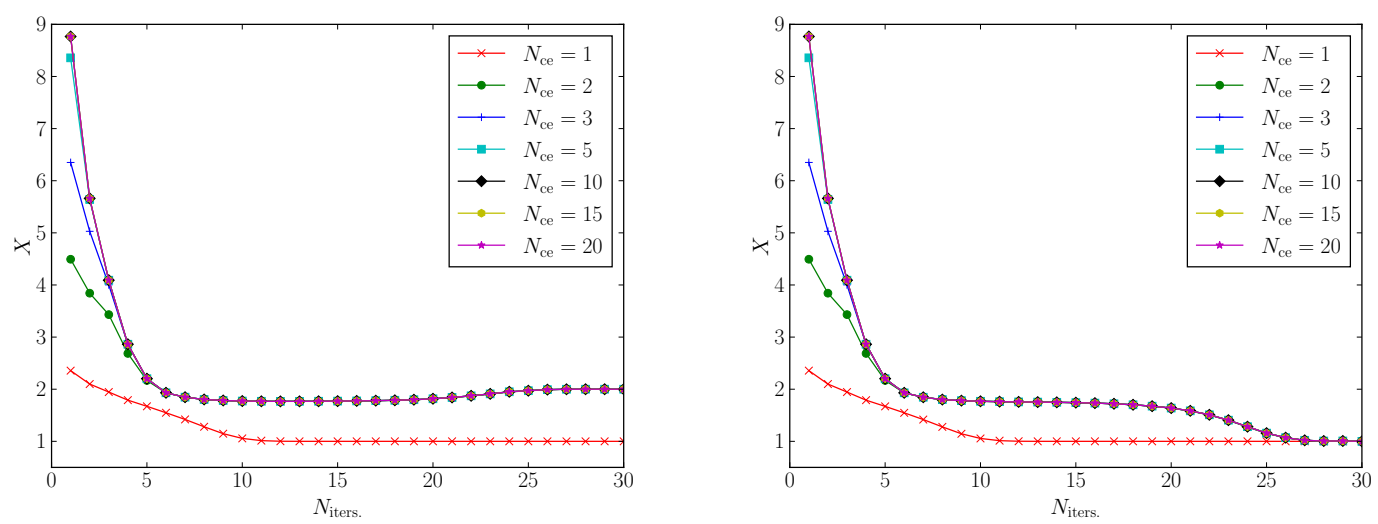

Figure 5: DTRG flows of $X$ for various values of $N_{\text {ce }}$ at $\beta=10.0$ with $D=16$. The left (right) panel shows the results for $\kappa=0.24130(\kappa=0.24135)$. All the flows for $N_{\mathrm{ce}} \geq 2$ converge to the same value.

up to $\beta=700.0$. A quadratic fit in $g$ with a data set $\beta=50.0,100.0,200.0,300.0,500.0,700.0$ is performed and our estimate of the continuum extrapolation is thus

$$
\left(\frac{m}{g}\right)_{c}=0.330(9)
$$

which agrees with the estimate by Byrnes et al. of $\left(\frac{m}{g}\right)_{c}=0.3335(2)$. The reason why ours is less accurate is thought to be that we employ the unimproved Wilson fermion which has $O(a)$ discretization error while Byrnes et al. employ the Kogut-Susskind fermion whose discretization error is $O\left(a^{2}\right)$. 


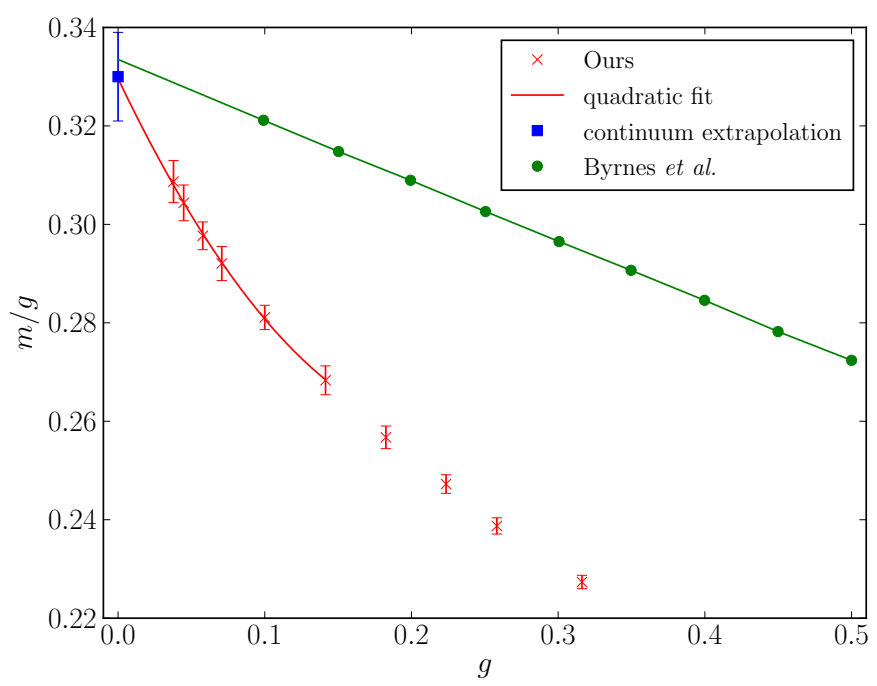

Figure 6: Critical value of $\frac{m}{g}$ as a function of $g$. The estimates by Byrnes et al. reported in Ref. [8] are also plotted. The red line indicates a quadratic fit in $g$ with a data set $\beta=50.0,100.0,200.0,300.0,500.0,700.0$ and the blue symbol is the continuum extrapolation derived from the fit.

\section{Acknowledgments}

Numerical calculations for the present work were mainly carried out using the computer facilities at Research Institute for Information Technology, Kyushu University and the HA-PACS computer under the "Collaborative Interdisciplinary Program" of Center for Computational Sciences, University of Tsukuba. Part of the computation was performed on the HOKUSAI-GW System at RIKEN Advanced Center for Computing and Communication.

\section{References}

[1] Y. Shimizu and Y. Kuramashi, Phys. Rev. D 90, 014508 (2014).

[2] Y. Shimizu and Y. Kuramashi, Phys. Rev. D 90, 074503 (2014).

[3] B. Dittrich, S. Mizera and S. Steinhaus, arXiv:1409.2407 [gr-qc].

[4] A. S. Hassan, M. Imachi and H. Yoneyama, Prog. Theor. Phys. 93, 161 (1995).

[5] A. S. Hassan, M. Imachi, N. Tsuzuki and H. Yoneyama, Prog. Theor. Phys. 94, 861 (1995).

[6] Y. Shimizu and Y. Kuramashi, in preparation .

[7] Z.-C. Gu and X.-G. Wen, Phys. Rev. B 80, 155131 (2009).

[8] T. M. R. Byrnes, P. Sriganesh, R. J. Bursill and C. J. Hamer, Phys. Rev. D 66, 013002 (2002). 